\title{
A rapid prototyping approach for custom training of autologous ear reconstruction
}

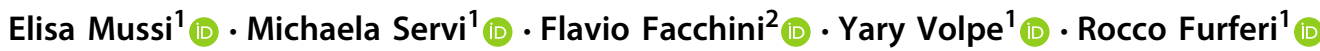

Received: 23 November 2020 / Accepted: 25 September 2021 / Published online: 8 October 2021

(c) The Author(s) 2021

\begin{abstract}
Autologous ear reconstruction is the preferred treatment in case of partial or total absence of the patient external ear. This kind of surgery can be really challenging since precise replication of complex three-dimensional structure of the ear is crucial to provide the patients with aesthetically consistent reconstructed anatomy. Therefore, the results strongly depends on the "artistic skills" of the surgeon who is in charge to carry out a three-dimensional sculpture, which resembles the shape of a normal ear. In this context, the definition of a preoperative planning and simulation process based on the patient's specific anatomy may help the surgeon in speeding up the ear reconstruction process and, at the same time, to obtain better results, thus allowing a superior surgical outcome. In the present work the main required features for performing an effective simulation of the ear reconstruction are identified and a strategy for their interactive design and customization is devised with the perspective of a semi-automatization of the procedure. In detail, the paper provides a framework which start from the acquisition of 3D data from both a healthy ear of the patient (or, if not available e.g. due to bilateral microtia of the ear of one of his parents or from a template) and of costal cartilage. Acquired 3D data are properly processed to define the anatomical elements of the ear and to find, using nesting-based algorithms, the costal cartilage portions to be used for carving the ear itself. Finally, 3D printing is used to create a mockup of the ear elements and a prototype of the ear to be reconstructed is created. Validated on a test case, the devised procedure demonstrate its effectiveness.
\end{abstract}

Keywords Autologous ear reconstruction $\cdot$ Microtia $\cdot$ Preoperative planning $\cdot$ Reverse engineering $\cdot$ Additive manufacturing

\section{Introduction}

Microtia is a congenital malformation characterized by the absence or deformation of the outer ear. This malformation occurs in 2.06 cases per 10,000 live births and may affect the size, orientation, shape and position of the outer ear. Three alternative treatments are used in clinical practice to address this physical malformation: (1) external silicone prosthesis [1, 2], (2) Medpor implants [3, 4], (3) reconstruction with autologous cartilage tissue extracted from the ribcage [5, 6].

The prosthetic approach is a non-permanent solution, while the alloplastic approach requires the insertion of a foreign body and is therefore more subjected to rejection.

Elisa Mussi

elisa.mussi@unifi.it

1 Department of Industrial Engineering, University of Florence, Florence, Italy

2 Meyer Children's Hospital, Florence, Italy
Accordingly, the autologous ear reconstruction (AER) surgery represents the preferred clinical treatment for restoring the anatomy of the ear as it is a definitive solution with high degree of integration, since autologous tissue harvested at costal level is used. The intervention foresees the realization of a 3D framework of the ear (Fig. 1b), obtained by carving and sculpting harvested patient's costal cartilages before the final implantation into a subcutaneous pocket in the auricular region. According to the technique proposed in [5], the auricular elements to be reconstructed are helix, antihelix, tragus-antitragus (Fig. 1a), plus a support base.

This procedure represents a challenge for plastic surgeons due to the complexity of the $3 \mathrm{D}$ geometry to be reproduced; in fact, the precise replication of this complex three-dimensional structure strongly relies on the own carving surgeon skills.

In particular, the most complex, yet crucial, phase consists of the reconstruction of the ear framework: using a good framework, it is possible to obtain a restoration of a fully 


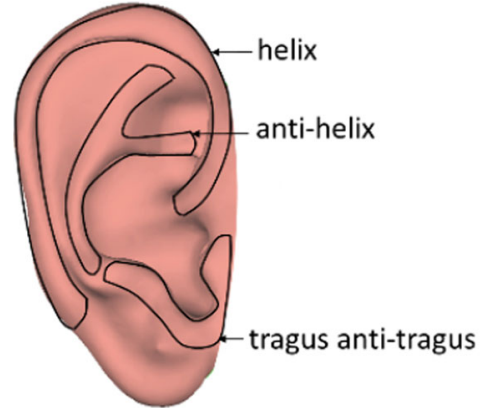

(a)

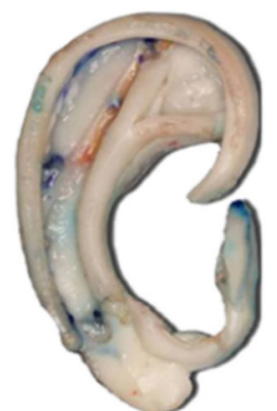

(b)
Fig. 1 a Auricular elements involved in the AER surgery and $\mathbf{b}$ ear framework

satisfactory, complete auricle, which is always the main goal of the patient and the plastic surgeon [6].

The correct creation of an ear framework can be carried out by using the following steps:

i. Study of the anatomy of the patient's healthy ear to understand involved volumes and dimensions. For this phase it has to be noticed that the ear cartilage of a child over eight years of age has very low variations in size as they grow up. Of course, if the patient has bilateral microtia, the healthy ear is not available. Therefore, the surgeon can refer to the ear of one of the patient parents (possibly uniformly scaled, if required). The key aspect of this process is to analyze the distinctive features of the reference ear in view of the successive 3D reconstruction of a framework that faithfully reproduces the anatomy. During this phase, an X-ray film is usually used to draw the contours of the healthy ear. This results in a 2D template, which can be mirrored and used (as a reference) in the reconstruction.

ii. Harvesting of individual elements from the cartilage. Typically, cartilage ranging from the sixth to the ninth rib is harvested from the patient.

iii. Sculpting and carving of the 3D framework. After the required elements are extracted from the cartilage, a manual process of sculpting, carving and suturing of the framework can be accomplished. The surgeon, using surgical instruments specifically designed for this procedure, performs this step. Once created, the framework is inserted into a subcutaneous pocket obtained in the auricular region. The complications that can occur at this level are the lack of available material or a particular conformation of the anatomy involved in the pathology.

Summing up, the critical aspects to correctly reconstruct the outer ear are the study of the anatomy of the patient's healthy ear to evaluate its volume and size; the visual isolation of the auricular elements involved in the surgery; the minimization of the removed cartilaginous tissue to create the 3D framework.

In order to achieve an aesthetically pleasing result, the reconstructed ear must look natural and sufficiently resemble the healthy ear. To this purpose, the surgeon should be specifically trained in both sculpting and carving to be able to reproduce the ear complex geometry.

Accordingly, the development of tools and methods for simulating the AER is considered a relevant issue since the surgeon can learn, test and improve several aspects of the surgical procedure prior to operate in the actual patient. In particular, the training aim is to develop an "aesthetic eye" able to identify proportions and harmonies and to increase technical skills. Another important advantage of the simulation is the possibility to familiarize with the patient's specific anatomy, which leads to a higher confidence of the surgeon in the surgery room and the optimization of the costal cartilageharvesting phase, minimizing the invasiveness of the surgery.

Nowadays, the simulation process can hinge on advanced Reverse Engineering (RE) and Additive Manufacturing (AM) tools, pushing the frontiers of medical simulation towards new patient-specific trainers [7]. Medical RE, in fact, allows collecting the patients' anatomical data manufactured with AM techniques capable of shaping geometries otherwise not obtainable by employing traditional techniques. Unfortunately, to date, the use of these technologies requires specialized engineers able to handle 3D geometries and 3D modelling with dedicated software. In the perspective of improving such a process, allowing the surgeon to simulate the intervention without the need of managing complex CAD-based data to perform the actual surgery on a mockup personalized on the patient, the introduction of interactive methods could be of great benefit.

For that reason, in the present paper the preoperative simulation and planning process is analyzed with the objective to develop an interactive procedure, exploiting RE and AM techniques that can be accessed directly by medical staff to create patient-specific simulators. The procedure is validated using a case study. The remainder of the paper is as follows: Sect. 2 describes the design process of custom simulators. In Sect. 3 a standard workflow for the production of simulators is proposed and analyzed. Finally, results and conclusions are drafted in Sect. 4.

\section{Pre-operative planning and simulation: medical requirements}

From the joint analysis carried out by engineers and surgeons on AER process, it emerged that the simulator must include two elements in order to consistently help surgeons to practice all the challenges of AER. In detail, such elements are the replica of the patient's cartilages (purple boxes in Fig. 2) 


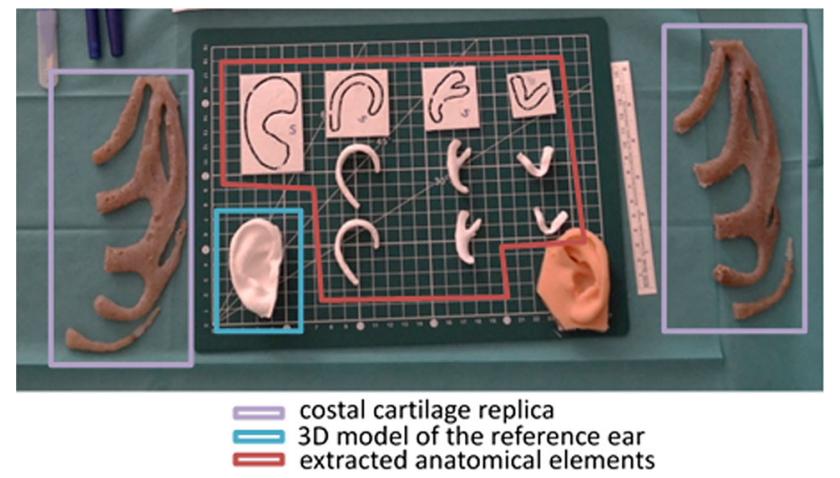

Fig. 2 Simulation table for pre-surgical planning

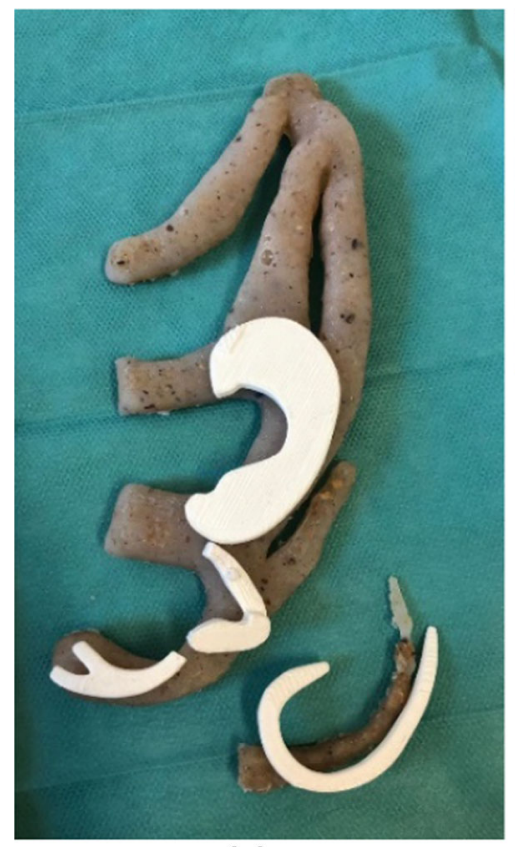

(a)

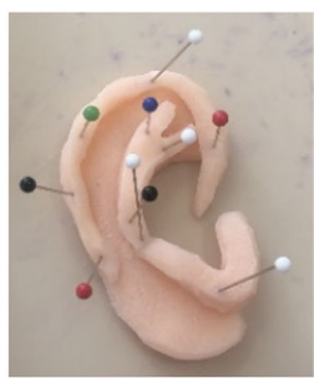

(b)

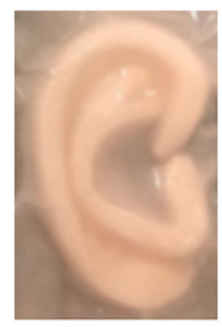

(c)
Fig. 3 AER simulation: a manual nesting, b reconstructed framework and $\mathbf{c}$ skin effect simulation on reconstructed framework

and the reproduction of the mirrored healthy ear (blue box in Fig. 2) and of the auricular elements (red box in Fig. 2).

In fact, the surgical simulation should begin with the analysis of the costal cartilage reproduction in order to identify the regions from which to extract the auricular elements to avoid waste of cartilage tissue. This step should be accomplished by evaluating the possible combinations of the elements on the cartilage replica, thus performing a manual nesting. Once the optimal positions are identified, the surgeon will be able to place the individual elements on the cartilage and manually draws their contours (see Fig. 3a). By following the traced silhouettes, the surgeon will engrave the elements and refine the details by sculpting and carving operations; successively the framework can be sutured (Fig. 3b).
Finally, the aesthetic results can be simulated, e.g. by using a $1 \mathrm{~mm}$ thick silicone pad to evaluate the effect of the skin over the framework [8]. Specifically, the framework should be placed between the pad and a suction device that, vacuuming the air, allows the silicone sheet to adhere to the framework (Fig. 3c).

The trainer for the AER simulation described above can be employed on a large scale only if the production phase is generalized and standardized, while maintaining a close adherence with the individual patient's anatomy. For this reason a strategy for customizing pre-operative planning and simulation has to be defined, as described below.

\section{Proposed strategy for custom preoperative planning}

With the aim of providing the surgeons with the consistent simulator for the AER described in Sect. 2, it is necessary to define a standard workflow that formalizes the main steps for the realization of patient-specific task trainers. Such a framework allows the physician to obtain the ear mockup in an interactive, autonomous way, using just a few inputs. Using the proposed method, the trainer can be manufactured in compliance with medical requirements without the involvement of RE and CAD modeling experts. This would represent on its own a significant step forward, since the availability of an easy-to-use tool allowing the medical staff to obtain in full autonomy the desired custom trainer leads to a significant reduction of both costs and timings.

The resulting workflow is shown in Fig. 4, in blue are depicted the steps leading to the elements required for the preoperative simulation (in red).

In brief, the workflow consists of two parallel processes: the first one leads to the creation of the auricular element's models and the second leads to the manufacturing of the costal cartilage replica.

In the first process (1a) the 3D model of the anatomy of the healthy ear is analyzed to identify the auricular elements involved in the simulation (2a).

As mentioned before, when a healthy ear cannot be extracted from the patient, the surgeon can also refer to the ear of one of the patient parents (possibly uniformly scaled, if required) or on an ear template.

The surgical templates are then manufactured using AM (3a). In parallel, in the second process, acquisitions obtained by means of diagnostic imaging techniques are used to reconstruct the $3 \mathrm{D}$ model of costal cartilages (1b). After a segmentation phase $(2 \mathrm{~b})$ the $3 \mathrm{D}$ modeling of molds suitable for the fabrication of silicone replicas can be carried out within a CAD environment ( $3 b)$.

Cartilage nesting (4) is performed with the two resulting elements, with the aim of minimizing the cartilage material to 


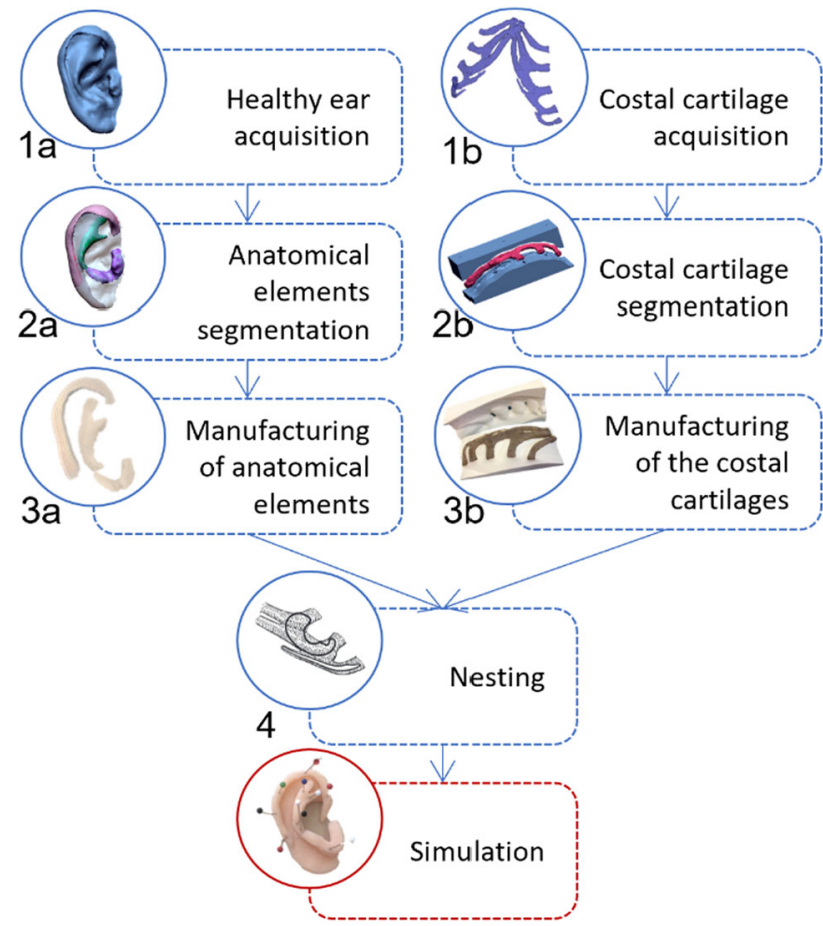

Fig. 4 Devised workflow for custom preoperative planning

be harvested from the patient. In the medical experience, the optimization of the cut was obtained experimentally by the surgeon directly with the physical models. The nesting phase can be considered as an integral part of the AER simulation.

If on the one hand the workflow phases can be performed manually by experts, on the other hand the automation of the processes can represent a great step forward for the distribution of the customized simulation of this type of surgery.

In order to make the production process of customized simulators semi-automatic and robust, each step introduced in the workflow are analyzed by investigating for each one three main aspects:

1. Identification of the best alternative among available methods in terms of results and timing.

2. Evaluation of the possibility of developing new methodologies.

3. Assessment of the feasibility of automating the step.

The workflow is detailed and analyzed according to the above-mentioned three aspects which are referred in the next sub-paragraphs with the number corresponding to the relative evaluated aspect.

Step 1a: Healthy ear acquisition

The workflow starts with the acquisition of the 3D geometry of the healthy ear. Referring to the three main aspects identified above, this step can be carried out in different ways, as described below:
1. In order not to subject the patient to invasive acquisition techniques, such as CT [9], this operation is usually performed with photogrammetry techniques or professional optical devices. Such techniques provide an accurate $3 \mathrm{D}$ reconstruction $(\sim 0.05 \mathrm{~mm})$ yet require scanning and post-processing times which are not suitable for clinical purposes.

2. The authors, to this end, evaluated the possibility to realize an external ear acquisition system using low-cost solutions [10] based on 3D scanning. Specifically, the reconstruction performances of photogrammetric techniques and depth camera approaches were compared. For this purpose, the Intel RealSense D415 [11] device was chosen on the basis of an exhaustive characterization. In fact, while the accuracy achievable with photogrammetric techniques is high, this requires an elaborate set-up and long reconstruction times. Quite the reverse, the acquisition using depth camera allows achieving acceptable accuracy for the application area. To assess both methods' accuracy, a ground truth model of an auricular anatomy was created using a professional scanner (Romer Absolute Arm [12], precision: $0.063 \mathrm{~mm}$ ). Figure 5 shows the results of the deviation from the reference model for the two tests.

In addition, this approach offers the advantage of shorter reconstruction times and the possibility of developing compact and manageable systems that can be used regularly in common clinical practice.

3. The semi-automatization of the process can be achieved by combining instantaneous acquisition configurations with the development of control software such as, for example, in [13]. With this idea, as in [], the authors intend to develop a system capable of acquiring and reconstructing the ear anatomy by combining an appropriate number of devices in an ad-hoc support, controlled through a simple user interface. This will allow the end-user to initiate the acquisition procedure via a simple input on the developed software.

\section{Step 2a: Anatomical elements segmentation}

In this step, the model of the entire ear is segmented to find the anatomical elements involved in the AER, for subsequent CAD modeling of surgical templates. Also for this step it is possible to draft three considerations, on the basis of the main aspects identified.

1. The manual detection of the contours of the ear elements can be performed following, for example, the study proposed by Iannarelli in [14]. On the other hand, it is desirable in order to streamline the production process, to automatize this phase. The literature, to date, does not offer solutions for the automatic recognition of the elements of the outer ear, starting from the 3D anatomy. 

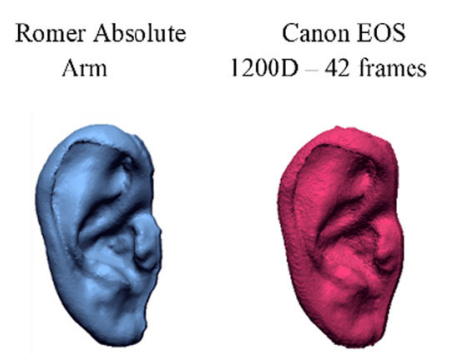

(a)
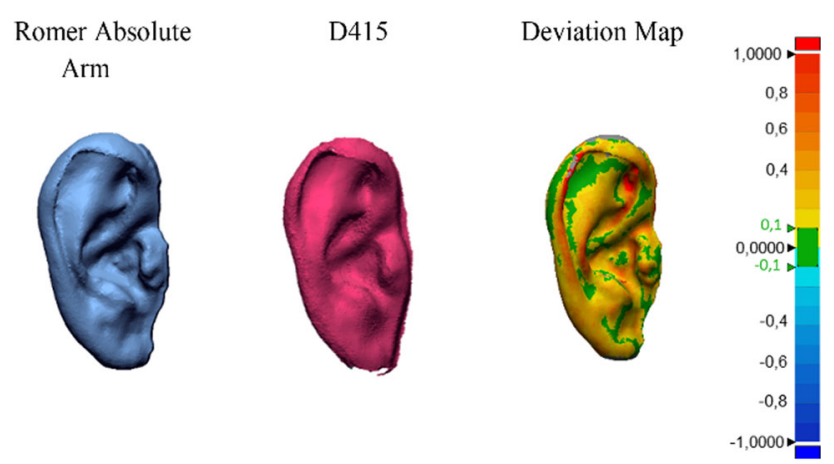

(b)

Fig. 5 Accuracy test for photogrammetry and depth camera acquisition

2. The spectrum of solutions that can be adopted and designed to approach this goal includes the use of threedimensional anatomical data, as well as $2 \mathrm{D}$ or hybrid solutions [15-17]. In addition, segmentation strategies, already used in the literature for different anatomical districts, can be investigated [18].

3. To this purpose, the authors are currently studying hybrid techniques to automatically segment the ear according to its main elements, possibly using interactive procedures. The investigated idea exploits information that can be gained from depth maps of the ear anatomy obtained by properly orienting the 3D model, in order to make clearly visible the auricular elements involved in the reconstruction. The depth-map base approach is a hybrid approach since the 2D image contains depth information defined by the 3D model. Figure 6a, b shows, respectively, an example of ear depth map and the model annotated (manually) which represents the desired segmentation. The authors intend to embed this process within the GUI mentioned above. This will allow the user to start the segmentation process by simply selecting the corresponding button.

\section{Step 3a: Manufacturing of anatomical elements}

The manufacturing phase, comprising CAD modeling and additive manufacturing, leads to the creation of physical

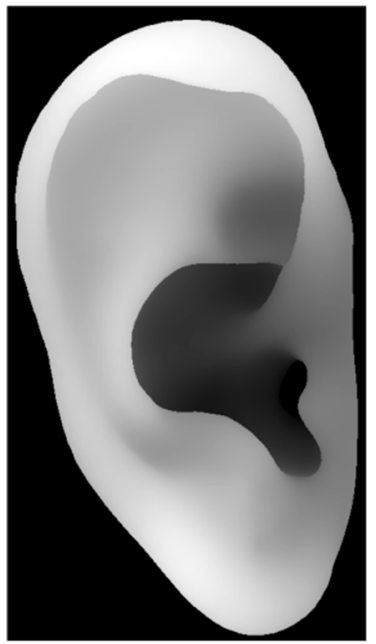

(a)

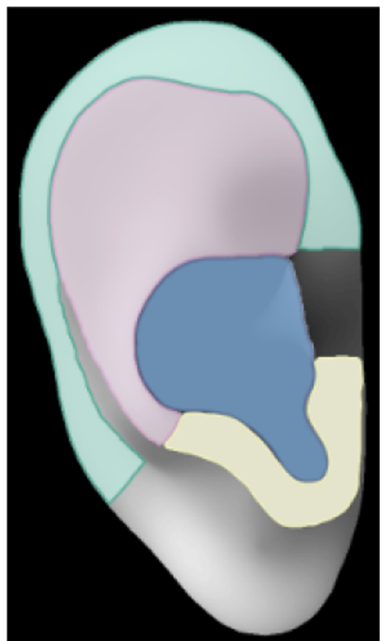

(b)
Fig. 6 Example of ear depth map (a) with corresponding elements segmentation (b)

mockups simplifying the actual anatomy of the ear. Referring to the three aspects the following considerations can be drafted:

1. The prototyped templates are considered by surgeons to be overly complex and not easily usable in the surgical phase for framework reconstruction, due to their millimetric details.

2. Accordingly, in collaboration with some plastic surgeons of the Meyer Children's Hospital, a process of simplification of the templates geometry were performed leading to the definition of a new design able to meet usability requirements while maintaining characteristics of the patient's anatomy [19]. The delineation of technical and clinical requirements of the surgical guides was the result of a close multidisciplinary collaboration between clinicians and engineers. The correct shape and functionality were identified through an iterative process of design and physical simulation during which the clinician tested the devised templates in the realization of the ear framework. Initially, according to the state-of-the-art procedures, the guides of each anatomical element were created following faithfully the original anatomy (see Fig. 7a). The shape of the surgical guides was subsequently defined as a simplification of each anatomical element's geometry. The simplification process consisted in the enhancement of certain edges and fundamental curves; this allows to obtain the desired aesthetic result during the surgical phase as the distinguishing features of the outer ear are reproduced and emphasized in view of the subsequent application of the skin layer (Fig. 7b).

3. In order to obtain a production process usable by hospital staff, the CAD modeling of the simplified templates will 

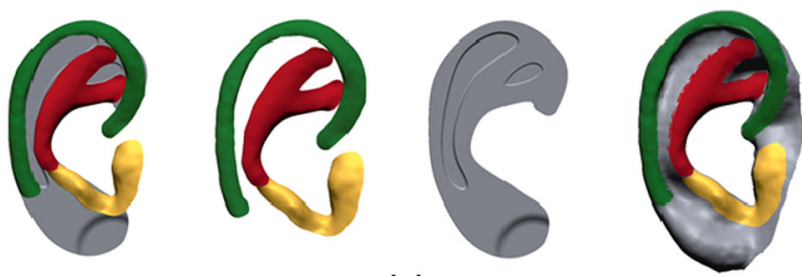

(a)
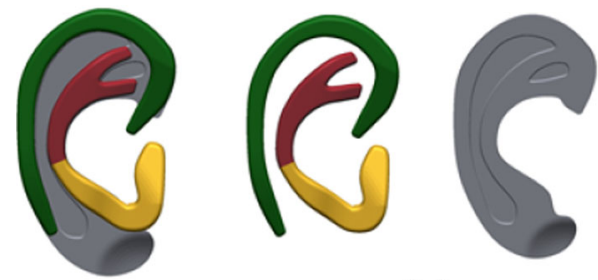

(b)

Fig. 7 Example of a surgical guides faithfully following the original anatomy (a) and created by simplify the original anatomy (b)

be managed through the dedicated GUI described above. Also in this case a button will be inserted to invoke in background the guides modeling routine.

\section{Step 1b: Costal cartilage acquisition}

To obtain the anatomy of the costal cartilage the standard acquisition process involves the use of diagnostic imaging techniques such as Computed Tomography scan (CT) and/or Magnetic Resonance Imaging (MRI), which through tomographic acquisition allow to reconstruct the model of the patient's internal anatomy. For this phase, the state of the art is hardly improvable since no external imaging systems can be used to retrieve the 3D model.

\section{Step 2b: Costal cartilage segmentation}

Starting from Digital Imaging and Communications in Medicine (DICOM) data, the costal cartilage 3D model is obtained. In DICOM data different tissues are characterized by different gray levels. Commonly the cartilages are isolated by manually setting appropriately chosen thresholds and with automatic segmentation algorithms IN MIMICS. Specifically, several studies implemented sufficiently accurate algorithms to extract costal cartilage tissue from $\mathrm{CT}$ without user input [20]. Also for this phase, the common technique adopted for segmentation is now a consolidated practice in medical literature.

Step 3b: Manufacturing of costal cartilage

This phase foresees the realization of the rib cartilage physical replica. Referring to the three aspects the following considerations can be enlisted.

1. The prototype of the ribs can be obtained through the production of moulds and the subsequent casting of silicone (or polyurethane) materials that are intended to simulate the mechanical behavior of the tissue, in order to make the simulation immersive and realistic [21].

2. To date no suitable materials have been identified to simultaneously enable an effective simulation of carving and sculpting operations and a real haptic return. In this perspective, the research can be directed towards additives for silicone materials or new direct additive production technologies (which would eliminate the need for moulds), to obtain tissue-mimicking materials.

3. In order to streamline and make the mould design phase independent from expert CAD modellers, the authors are investigating the possibility of automating this step. For this purpose, it is necessary to define a routine of CAD operations that can systematically lead to the modeling of the mould independently of the inter-individual variability of the anatomy of interest. It will be necessary to use programmable CAD modeling software or opensource CAD libraries such as OpenCascade [22] which is programmable in python or $\mathrm{C}++$ languages. As for the guide modelling task a dedicated button will be inserted to invoke in background the $\mathrm{CAD}$ modeling routine.

\section{Step 4: Nesting}

In phase 4 the surgeon is provided with the 3D model of the ear and the reproduction of the rib cartilage, thus can study the optimal cutting of the anatomical elements in order to minimize the cartilaginous tissue to be harvested during surgery. The goal is to nest the elements such to identify the necessary portion of cartilage that will be removed from the patient for the $3 \mathrm{D}$ reconstruction of the framework. The three aspects are evaluated:

1. To date, this operation is manually performed by the surgeon by empirically testing the templates positions on the cartilage.

2. Wide prospects of improvement arise with the idea of using $2 \mathrm{D}$ nesting algorithms available in literature or with a view of implementing ad-hoc methods that consider 3D characteristics of the involved anatomies. While these techniques are now widely investigated in areas such as fabric cutting [23] or storage problems [24], they are not yet explored in the field of autologous ear reconstruction. For this purpose algorithms involving irregular containers can be considered and which take into account the presence of any internal areas not belonging to the container while optimizing the positioning of non-regular objects such as $[25,26]$.

3. Automatic solutions in this direction can lead to exempt the physician from an empirical phase that besides being time-consuming may lead to sub-optimal solutions. Also for this final step a dedicated button can be inserted to invoke in background the $\mathrm{CAD}$ modeling routine. 


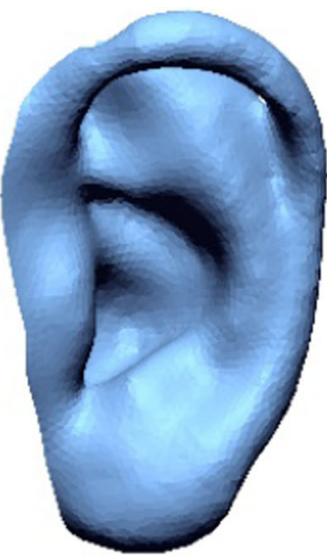

(a)

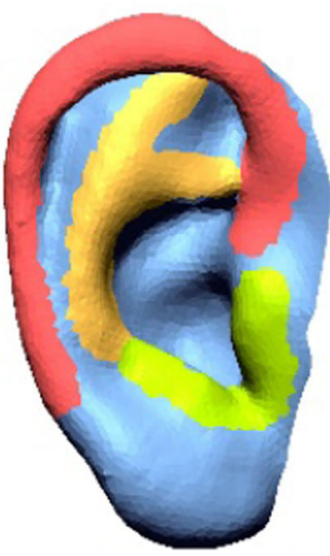

(b)

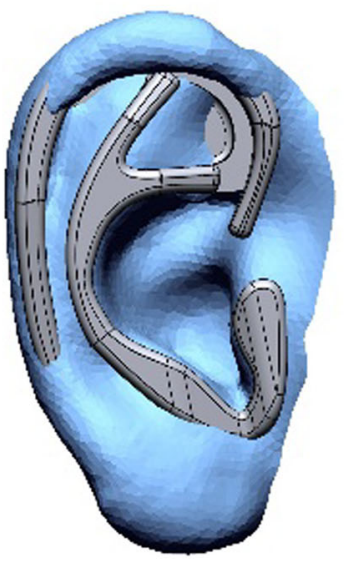

(c)
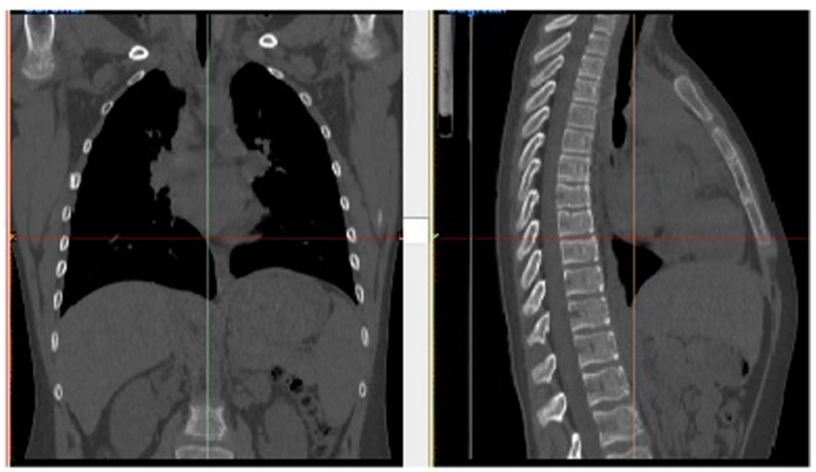

(a)

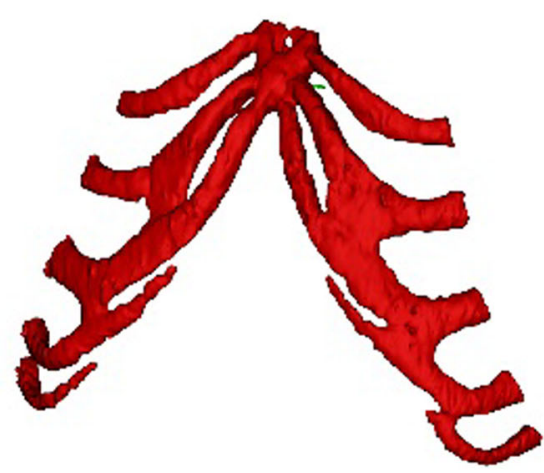

(b)

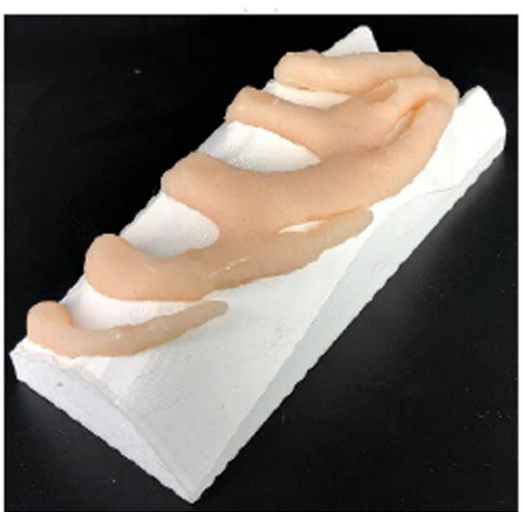

(c)
Fig. 8 Phases 1a, 2a and 3a of the devised workflow applied to a case study

\section{Validation of the proposed framework}

In this section is reported a case study with the intent to validate the framework identified for the realization of custom trainers, detailed in the Sect. 3. The case study refers to a 14year-old pediatric patient of the Meyer Children's Hospital (Firenze, Italy) affected by unilateral microtia on right ear.

The patient's healthy ear was acquired with the Intel RealSense D415 device. Figure 8a shows the obtained 3D model (Step 1a). Subsequently, the anatomical elements were manually segmented (Step 2a, Fig. 8b) and the surgical guides were modeled (Fig. 8c). For the surgical guides CAD modeling, the process of simplification of the anatomy described above was followed. The medical devices were then manufactured with additive techniques using ABS-M30i [27] (Step $3 a)$.

To model the costal cartilage, the patient underwent a CT scan (Step 1b, Fig. 9a) which was then processed using thresholding methods to obtain desired geometry (Step $2 b$, 


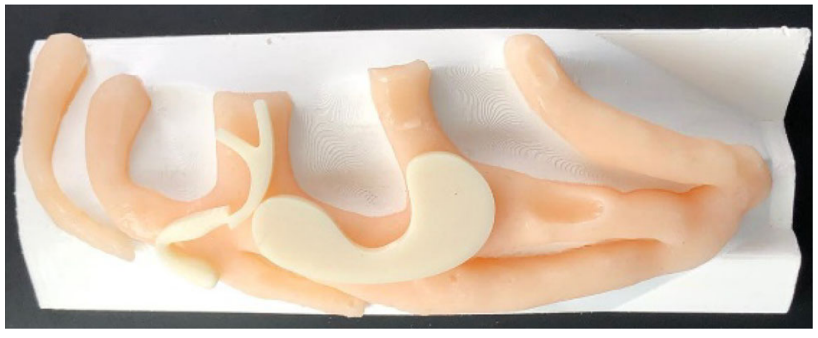

Fig. 10 Phase 4 of the devised workflow applied to a case study

in literature use these techniques focusing on specific steps of the process described above (e.g. ear acquisition [29] or the modelling of surgical guides $[30,31]$ ), however to date there is no comprehensive tool capable of assisting the surgeon, not only in the operating room with custom medical devices, but also in the autonomous production of these devices, as well as pre-operative simulators. For this reason, the results obtained from this case study are not comparable to other research but constitute an innovation in the field.

\section{Conclusions}

The workflow for the creation of patient-specific AER trainers was proposed in this paper. The definition of a standard workflow allows splitting the process into a number of steps, which lead from 3D data acquisition of both ear and rib cartilages to the definition of prototypes to be used for intervention planning and simulation. For each step, three different aspects were drafted, covering the identification of the best alternative among available methods in terms of results and timing, the evaluation new methodologies to be possibly used to streamline the process and the assessment of possible automatization of the process.

This lays the foundation for the automation of some processes with the aim of obtaining an interactive procedure allowing the physician to autonomously generate a patientspecific AER trainer.

The semi-automatization of the workflow is currently under evaluation. Some steps have been standardized such as the use of diagnostic imaging to obtain geometric information about costal cartilage; others have been addressed and partially solved by the authors such as the acquisition of the outer ear anatomy and the modeling of surgical guides. With regard to the remaining phases, the authors have identified possible strategies and tools to make the process fully or partially automatic. The semi-automation of the entire procedure will lead to the development of a dedicated software able to integrate the described processes and make them available to the medical staff through an intuitive graphical interface that will require user interaction to trigger the steps of the process and insert some anatomical repère interactively.
The result of a future automatization represents a key point, being AER a very complex surgery for which the introduction of the patient-specific preoperative simulation would significantly mitigate the challenge even for the most experienced surgeons. Thus, the semi-automatization of the simulator production process would facilitate training in a hospital environment. Within this context, this human-machine interaction allows realizing a semi-automatic process, which enables, on the one hand, to overcome some limits of the state of the art and, on the other hand, to obtain a trainer that satisfies the medical requirements.

Funding Open access funding provided by Università degli Studi di Firenze within the CRUI-CARE Agreement.

Open Access This article is licensed under a Creative Commons Attribution 4.0 International License, which permits use, sharing, adaptation, distribution and reproduction in any medium or format, as long as you give appropriate credit to the original author(s) and the source, provide a link to the Creative Commons licence, and indicate if changes were made. The images or other third party material in this article are included in the article's Creative Commons licence, unless indicated otherwise in a credit line to the material. If material is not included in the article's Creative Commons licence and your intended use is not permitted by statutory regulation or exceeds the permitted use, you will need to obtain permission directly from the copyright holder. To view a copy of this licence, visit http://creativecomm ons.org/licenses/by/4.0/.

\section{References}

1. Federspil, P.A.: Auricular prostheses in microtia. Fac. Plast. Surg. Clin. N. Am. 26, 97-104 (2018). https://doi.org/10.1016/J.FSC.20 17.09.007

2. Artioli, B.O., Kunkel, M.E., Mestanza, S.N.: Feasibility study of a methodology using additive manufacture to produce silicone ear prostheses. IFMBE Proc. 68, 211-215 (2019). https://doi.org/10.1 007/978-981-10-9023-3_38

3. Williams, J.D., Romo, T., Sclafani, A.P., Cho, H.: Porous highdensity polyethylene implants in auricular reconstruction. Arch. Otolaryngol. Head. Neck Surg. 123, 578-583 (1997). https://doi. org/10.1001/ARCHOTOL.1997.01900060020003

4. Owen, S., Wang, T., Stephan, S.: Alloplastic reconstruction of the microtic ear. Oper. Tech. Otolaryngol. Neck Surg. 28, 97-104 (2017). https://doi.org/10.1016/J.OTOT.2017.03.007

5. Nagata, S.: A new method of total reconstruction of the auricle for microtia. Plast. Reconstr. Surg. 92, 187-201 (1993). https://doi. org/10.1097/00006534-199308000-00001

6. Storck, K., Staudenmaier, R., Buchberger, M., Strenger, T., Kreutzer, K., Von Bomhard, A., Stark, T.: Total reconstruction of the auricle: our experiences on indications and recent techniques. https://pubmed.ncbi.nlm.nih.gov/24822198/ (2014)

7. Giannatsis, J., Dedoussis, V.: Additive fabrication technologies applied to medicine and health care: a review. Int. J. Adv. Manuf. Technol. 40, 116-127 (2009). https://doi.org/10.1007/s00170-0071308-1

8. Firmin, F., Marchac, A.: A novel algorithm for autologous ear reconstruction. Semin. Plast. Surg. 25, 257-264 (2011). https:// doi.org/10.1055/s-0031-1288917

9. Nuseir, A., Hatamleh, M., Watson, J., Al-Wahadni, A.M., Alzoubi, F., Murad, M.: Improved construction of auricular prosthesis by 
digital technologies. J. Craniofac. Surg. 26, e502-e505 (2015). https://doi.org/10.1097/SCS.0000000000002012

10. Furferi, R., Mussi, E., Servi, M., Uccheddu, F., Volpe, Y., Facchini, F.: 3D acquisition of the ear anatomy: a low-cost set up suitable for the clinical practice. In: IFMBE Proceedings, pp. 669-678. Springer (2020)

11. Keselman, L., Woodfill, J.I., Grunnet-Jepsen, A., Bhowmik, A.: Intel R RealSense TM Stereoscopic Depth Cameras

12. ROMER Absolute Arm I Hexagon Manufacturing Intelligence. https://www.hexagonmi.com/products/portable-measuring-arms/ romer-absolute-arm

13. Buonamici, F., Furferi, R., Governi, L., Lazzeri, S., Mc Greevy, K.S., Servi, M., Talanti, E., Uccheddu, F., Volpe, Y.: A practical methodology for computer aided design of custom 3D printable casts for wrist fractures. Vis. Comput. (2019). https://doi.org/10.1 007/s00371-018-01624-z

14. Iannarelli, A.: Ear identification (1964)

15. Alemran, A., Rahmatullah, B. binti, Hadi, A.: Systematic review on ear identification (2018)

16. Emeršič, Ž, Štruc, V., Peer, P.: Ear recognition: more than a survey. Neurocomputing 255, 26-39 (2017). https://doi.org/10.1016/ j.neucom.2016.08.139

17. Lei, J., You, X., Abdel-Mottaleb, M.: Automatic ear landmark localization, segmentation, and pose classification in range images. IEEE Trans. Syst. Man Cybern. Syst. 46, 165-176 (2016). https:// doi.org/10.1109/TSMC.2015.2452892

18. Saleh, A., Rashwan, H.A., Abdel-Nasser, M., Singh, V.K., Abdulwahab, S., Mostafa Kamal Sarker, M., Garcia, M.A., Puig, D.: Finseg: finger parts semantic segmentation using multi-scale feature maps aggregation of FCN. In: VISIGRAPP 2019-Proceedings of the 14th International Joint Conference on Computer Vision, Imaging and Computer Graphics Theory and Applications, pp. 77-84. SciTePress (2019)

19. Facchini, F., Morabito, A., Buonamici, F., Mussi, E., Servi, M., Volpe, Y.: Autologous Ear Reconstruction: Towards a Semiautomatic CAD-Based Procedure for 3D Printable Surgical Guides. Presented at the May 25 (2020)

20. Barbosa, D., Queirós, S., Rodrigues, N., Correia-Pinto, J., Vilaça, J.: Semi-automatic 3D segmentation of costal cartilage in CT data from Pectus Excavatum patients. In: Ourselin, S., Styner, M.A. (eds.) Medical Imaging. p. 94133M. SPIE, Bellingham (2015)
21. Rossman, A.H., Catenacci, M., Zhao, C., Sikaria, D., Knudsen, J.E., Dawes, D., Gehm, M.E., Samei, E., Wiley, B.J., Lo, J.Y.: Three-dimensionally-printed anthropomorphic physical phantom for mammography and digital breast tomosynthesis with custom materials, lesions, and uniform quality control region. J. Med. Imaging 6, 1 (2019). https://doi.org/10.1117/1.jmi.6.2.021604

22. Open Cascade-software development company. https://www. opencascade.com/

23. Chen, B., Chou, T.W.: Compaction of woven-fabric preforms: nesting and multi-layer deformation. In: Composites Science and Technology, pp. 2223-2231. Elsevier (2000)

24. Jones, D.R.: A fully general, exact algorithm for nesting irregular shapes. J. Glob. Optim. 59, 367-404 (2014). https://doi.org/10.10 07/s10898-013-0129-z

25. Ramesh Babu, A., Ramesh Babu, N.: A generic approach for nesting of 2-D parts in 2-D sheets using genetic and heuristic algorithms. CAD Comput. Aided Des. 33, 879-891 (2001). https://doi. org/10.1016/S0010-4485(00)00112-3

26. Bennell, J.A., Oliveira, J.F.: The geometry of nesting problems: a tutorial. Eur. J. Oper. Res. 184, 397-415 (2008). https://doi.org/1 0.1016/j.ejor.2006.11.038

27. ABS-M30: A High Strength 3D Printing Material I Stratasys

28. 3D Printers, 3D Scanning, Software, Manufacturing and Healthcare Services I 3D Systems. https://www.3dsystems.com/

29. Ross, M.T., Cruz, R., Brooks-Richards, T.L., Hafner, L.M., Powell, S.K., Woodruff, M.A.: Comparison of three-dimensional surface scanning techniques for capturing the external ear. Virtual Phys. Prototyp. 13, 255-265 (2018). https://doi.org/10.1080/17452759. 2018.1493803

30. Flores, R.L., Liss, H., Raffaelli, S., Humayun, A., Khouri, K.S., Coelho, P.G., Witek, L.: The technique for 3D printing patientspecific models for auricular reconstruction. J. Cranio-Maxillofac. Surg. 45, 937-943 (2017). https://doi.org/10.1016/j.jcms.2017.03. 022

31. Witek, L., Khouri, K.S., Coelho, P.G., Flores, R.L.: Patient-specific 3D models for autogenous ear reconstruction. Surg Glob Open 4, e1093 (2016)

Publisher's Note Springer Nature remains neutral with regard to jurisdictional claims in published maps and institutional affiliations. 